\title{
APROXIMACIÓN A LA VALORACIÓN DE OPCIONES BAJO EL ANÁLISIS DE LA TEORÍA DE JUEGOS
}

\author{
Humberto Banda Ortiz*
}

Departamento de Administración y Finanzas

Tecnológico de Monterrey, Campus Santa Fe

\section{Orestes Gámez Díaz**}

Departamento de Administración y Finanzas

Tecnológico de Monterrey, Campus Santa Fe

(Recibido 6 de diciembre 2004, aceptado 25 de febrero. 2005)

\section{Resumen}

Desde los pioneros trabajos de Black y Scholes (1973) y Merton (1973), el campo de la valuación de activos derivados ha tenido un vertiginoso crecimiento. 'Los estudios posteriores a estos autores han permitido el desarrollo de lo que hoy se conoce como Option Pricing. El foco central del presente artículo es la utilización de la Teoría de Juegos como una herramienta de análisis, para la introspección y entendimiento de situaciones óptimas y de equilibrio en aplicaciones de negocios. Este estudio incluye reflexiones teóricas y empíricas, intentando mostrar el potencial del avance logrado por las finanzas en los últimos años.

\section{Abstract}

From the works of Black and Scholes (1973) and Merton (1973), the valuation of derivatives has had a vertiginous growth. The innumerable studies after these authors have allowed the development in the field of Option Pricing. The central goal of the present article is the use of the Theory of Games like an analytical tool for the introspection and understanding of optimal situations and balance in applications of businesses. This study includes theoretical and empirical reflections that show the advance obtained by the finances in the recent years.

Clasificación JEL: C70, G12, G13, M52

Palabras clave: Opciones, Teoría de Juegos, Valoración

* Profesor-Investigador y Coordinador de la Academia de Finanzas, Tecnológico de Monterrey, Campus Santa Fe. Av. Carlos Lazo No. 100. Col. Santa Fe, c. p. 01389. México, D.F. Teléfono (55) 9177-8000 (ext. 7781). Correo electrónico: humberto.banda@itesm.mx

** Profesor-Investigador y Dircctor del Departamento de Administración y Finanzas, Tecnológico de Monterrey, Campus Santa Fe. Av. Carlos Lazo No. 100. Col. Santa Fe, c. p. 01389. México, D.F. Correo electrónico: ogamez@itesm.mx 


\section{Introducción}

Desde los pioneros trabajos de Black y Scholes (1973) y Merton (1973), el campo de la valuación de activos derivados ha tenido un vertiginoso crecimiento. Los innumerables estudios posteriores a estos autores han permitido el desarrollo de lo que hoy se conoce como Option Pricing (cuya traducción más cercana al castellano podría ser "Valoración de Opciones"). ${ }^{1}$

Existe una variada gama de estudios donde se demuestra que los pasivos de las empresas pueden ser examinados como opciones respecto del valor de la misma compañía. La teoría de opciones nos ayuda a evaluar un componente muy importante que está presente en algunas de las decisiones de inversión.

No obstante, la incorporación de la teoría de opciones en forma ingenua podría dejar de un lado otro componente clave: el ambiente competitivo, ya que muchas decisiones de inversión están condicionadas a las reacciones de nuestros competidores. A la vez, las decisiones de nuestros competidores pueden depender de nuestras propias decisiones. Es aquí donde la Teoría de Juegos puede ayudarnos a definir, no sólo cuál es la mejor decisión de inversión en un momento dado, sino también a concebir una estrategia de inversión condicionada a lo que hagan nuestros competidores.

\section{Aspectos metodológicos}

Beneficiado por la maleabilidad analítica de modelos de tiempo continuo y lo cercano de las formas de modelos de valuación de derivados, Ziegler (1999) muestra como el conjunto de valuación de opciones pueden ser aplicados a situaciones donde agentes económicos interactúan estratégicamente.

La intuición básica del método utilizado por Ziegler (1999) es separar el problema de valoración de payoffs del análisis de interacciones estratégicas, logrando su objetivo en forma concisa; con lo cual algunas aplicaciones de este método son:

- La valoración de derechos contingentes cuando el comportamiento estratégico de los agentes económicos es posible,

- El análisis de incentivos de algunos convenios contractuales financieros comunes y

- El diseño de contratos de incentivo que se dirigen a resolver conflictos de intereses entre agentes económicos.

Por otra parte, los elementos esenciales para el análisis planteado son los siguientes: la existencia de Common knowledge para los participantes del juego, información perfecta conocida por los jugadores, y resolución del juego con el procedimiento de inducción hacia atrás. Además, cabe recordar que en un juego de información perfecta, los jugadores se mueven en un solo momento y cada uno de ellos conoce todos los momentos previamente cuando toma su decisión. ${ }^{2}$

1 Para una revisión resumida, conceptual y con ejercicios de opciones, recomendamos a Lamothe (1993), Constantinides y Malliaris (2001) y Fernández (1996). Para una revisión más profunda y analítica de este tema, recomendamos Chriss (1996), Briys. Minh, Bellatah y de Varenne (1998) y Schwartz y Smith (1997).

2 Para una revisión completa, descriptiva y profunda de Teoría de Juegos. recomendamos revisar Gibbons (1993) y Tirole (1990) en su capítulo II. 
La idea de que cada subjuego sería reemplazado con su pago de equilibrio es llamado "perfección en subjuegos", que es un criterio para eliminar los equilibrios de Nash que se basan en amenazas o promesas que no deberían ser creídas. Nótese que en un juego finito de información perfecta, la inducción hacia atrás y la perfección en subjuegos son equivalentes.

\section{Descripción del modelo}

Se asume que el juego sigue un Movimiento Geométrico Browniano descrito por $\mathrm{d} S=\mu S \mathrm{~d} t+\sigma S \overline{\mathrm{d} z}$, donde $S$ es el activo subyacente, $\mu$ es la tendencia (retorno instantáneo esperado), $\sigma$ es la desviación estándar instantánea del proceso, $\overline{\mathrm{d} z}$ es el incremento de un proceso estándar de Wiener y $t$ es el tiempo.

Bajo estas condiciones, si el valor del activo contingente se denota por $F(S, t)$, entonces, la función $F$ debería satisfacer la siguiente ecuación diferencial parcial lineal

$$
1 / 2 \sigma^{2} S^{2} F_{s s}+r(S-a)+F_{t}-r F+b=0 .
$$

Con $F$ en derivadas parciales, donde $r$ es la tasa libre de riesgo, a es el payout del tenedor del activo subyacente por unidad de tiempo, $b$ es el payout del tenedor del activo contingente por unidad de tiempo que debería satisfacer esta relación, difiriendo sólo de sus condiciones límites.

\subsection{El método de análisis de opciones con teoría de juegos}

La esencia del método puede ser resumido como un proceso de 3 pasos:

1. El juego entre jugadores es definido: estrategias, alternativas y payoffs.

2. Los payoffs futuros inciertos de los jugadores son valorados.

3. El juego es resuelto por estrategias óptimas de los jugadores usando la inducción hacia atrás o perfección en subjuegos.

Este análisis reemplaza a la maximización de la utilidad esperada, típica de los modelos clásicos, con la maximización del valor de una opción. Esto genera un valor libre de arbitraje del payoff al jugador y puede ser considerado como una variable proxy para la utilidad esperada. Una gran ventaja de esta mecánica, es que incluye en su análisis el concepto del valor del dinero en el tiempo y el del precio del riesgo.

De esta manera, si un jugador 1 elige la estrategia $A$, entonces, a éste le sucede un jugador 2 que elige la estrategia $B$. Ambas estrategias, junto al valor futuro del estado de la variable $S$, determinan los payoffs de cada uno de los jugadores. Por lo tanto, $G(A, B, S)$ y $H(A, B, S)$ denotan el valor corriente de libre-arbitraje de los payoffs de los jugadores 1 y 2 , respectivamente. Luego, las estrategias consisten en elegir uno de los parámetros de la ecuación diferencial o condiciones límites para maximizar el valor de su payoffs.

En el último escenario del juego, el jugador 2 elige aquella estrategia $B$ que maximiza el valor de su payoff esperado $H(A, B, S)$, es decir, $\frac{\partial H(A, B, S)}{\partial B}=0$ donde $B$ no es una solución límite.

Esta condición de primer orden puede ser resuelta para generar una estrategia óptima $\bar{B}=\bar{B}(A, S)$ y la anticipación del jugador 1 a esta decisión del 
jugador 2 se puede expresar como:

$$
\frac{\partial G(A, \bar{B}, S)}{\partial A}=\frac{\partial G(A, \bar{B}, S)}{\partial A}+\underbrace{\frac{\partial G(A, \bar{B}, S)}{\partial B} \times \frac{\partial B}{\partial A}}_{\text {Efecto Indirecto }}=0 .
$$

El efecto indirecto se puede analizar como un proceso de inducción hacia atrás, donde refleja el efecto indirecto del jugador 2 y su estrategia elegida que depende de la estrategia del jugador 2. De este modo se genera una estrategia óptima $\bar{A}=\bar{A}(S)$.

\section{Determinando el precio de una opción "Put Perpetua"}

\subsection{Supuestos adicionales y metodología de resolución}

Adicional a los elementos ya expuestos que son necesarios para la formalidad de estos modelos, es necesaria la existencia de mercados competitivos, donde se realiza la venta de una opción "Put Perpetua" de un activo subyacente $S$ con precio de ejercicio $X$.

Paso 1. Estructura del juego:

$\mathrm{Al}$ inicio, el intermediario vende la opción al inversionista a un precio cierto $P_{\infty}$, entonces, el inversionista posee la opción hasta que decide ejercerla, donde $\bar{S}$ es su estrategia de ejercicio óptima. Al ejercer la opción, el payoff del inversionista iguala la diferencia (positiva) entre el precio del ejercicio $X$ y el valor corriente del activo subyacente $\bar{S} \equiv \operatorname{Max}[0 ;(X-\bar{S})]$

Figura 1.

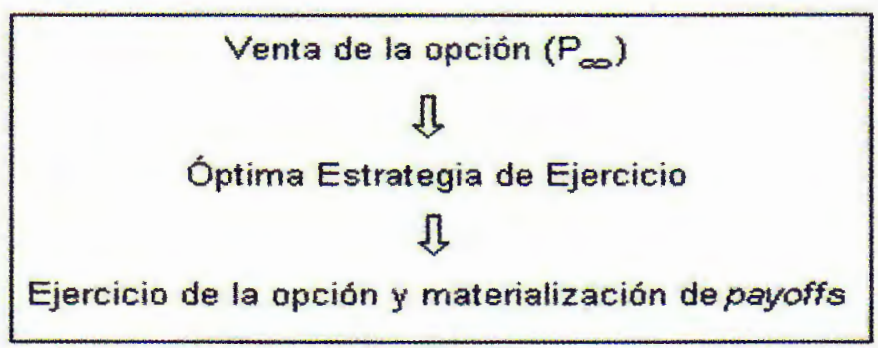

Paso 2. Valoración de la opción $\left(P_{\infty}(S)\right)$ :

La valoración se desprende de Merton (1973), donde $1 / 2 \sigma^{2} S^{2} P_{\infty}+r S P_{\infty}-$ $r P_{\infty}=0$ queda sujeto a las condiciones límites $P_{\infty}(\infty)=0$ y $P_{\infty}(\bar{S})=(X-\bar{S})$ y la solución general resulta ser $P_{\infty}(S)=a_{1} S+a_{2} S^{-\gamma}$, con $\gamma=2 r / \sigma^{2}$. Todo esto implica que $P_{\infty}(\bar{S})=a_{2} S^{-\gamma}$ y $a_{2}=(X-\bar{S}) \bar{S}^{\gamma}$. Por lo tanto, el valor de una opción put perpetua para una estrategia de ejercicio óptima queda dado por $P_{\infty}(S)=(X-\bar{S})\left(\bar{S}^{\gamma} S^{-\gamma}\right)$, lo que es igual a $(X-\bar{S})(S / \bar{S})^{-\gamma}$.

Paso 3. Resolviendo el juego:

Dado el paso 2 y como la estrategia $\bar{S}$ es aún desconocida para resolver el juego, se utilizan dos aproximaciones para llegar a la resolución final: "Smooth 
Pasting" y "Maximización del Valor $\bar{S}$ ". A continuación se presentan cómo ambas llegan a conclusiones similares.

a) "Smooth Pasting". Para esta aplicación se requiere de la condición "Smooth Pasting", que es: $\partial P_{\infty}(S) /\left.\partial S\right|_{S=\bar{S}}=\partial \bar{P}_{\infty} / \partial \bar{S}$, donde $\bar{P}_{\infty}$ es el valor" de la opción ejercida como especificación de la condición límite $\bar{P}_{\infty}=(X-\bar{S})$. De esta manera dicha condición en $P_{\infty}(S)$ toma el valor -1 , ya que $P_{\infty}(\bar{S})=$ $(X-\bar{S})$ y así se obtendría la valorización para $\bar{S}=\left[\frac{\gamma}{1+\gamma}\right] X$.

b)Maximización del valor estratégico de ejercicio $(\bar{S})$. Esta aproximación se realiza a través de la derivación de la función $P_{\infty}(S)$ del paso 2. Es decir, en

$$
\frac{\partial P_{\infty}(S)}{\partial \bar{S}}=\frac{\partial\left[(X-\bar{S})(S / \bar{S})^{-\gamma}\right]}{\partial \bar{S}}=0
$$

se obtiene que $\bar{S}=\gamma(X-\bar{S})$, lo que es igual a $\bar{S}=\left[\frac{\gamma}{1+\gamma}\right] X$ que es la misma solución que se obtuvo en el inciso a).

Esta coincidencia fue ratificada y demostrada por Merton (1973), de tal manera que en un punto óptimo $\bar{x}^{*}$, la función $f$ se maximiza en $\frac{\partial h}{\partial x}=\left.\frac{\partial f}{\partial x}\right|_{x=\bar{x}}$, donde $h$ es una función de $\bar{x}$ y $\frac{\partial^{2} f}{\partial \bar{x}^{2}}<0$. Entonces, la relación entre ambas aproximaciones se encuentra en los diferentes supuestos de los comportamientos de los agentes.

\subsection{La solución dada por Ziegler}

La conclusión a que llega Ziegler (1999) respecto de encontrar el precio intermedio de la opción, está en que el intermediario (el option writer) puede esperar anticiparse al inversionista en el punto donde él ejercerá el precio óptimo. Por lo tanto, él deberá preguntarse por un precio igual al valor de la opción óptimamente ejercido por el tenedor del derecho (el precio dado en el paso 2) con la estrategia dada en el paso 3. Con esto se llega a que el precio esperado en el mercado es

$$
P_{\infty}(S)=(X-\bar{S})\left(\frac{S}{\bar{S}}\right)^{-\gamma}
$$

o bien

$$
P_{\infty}(S)=\left(\frac{X}{1+\gamma}\right)\left(\frac{(1+\gamma) S}{\gamma X}\right)^{-\gamma} .
$$

\section{Otras aplicaciones}

\section{1 "Real Options"}

Una de las más proliferas aplicaciones de la valuación de opciones en el mundo no académico ha sido el de las "Real Options". Particularmente, uno de los campos que ha tomado mayor desarrollo es el de proyectos de ingeniería y de prospección. En este sentido, sería útil y natural la utilización de la teoría de juegos en este terreno. Esto es así ya que la inducción hacia atrás sería una poderosísina arma para la explicación de este tipo de análisis. 
Una aplicación de "Real Options" más cercana al campo de la administración, podemos encontrarla en la evaluación de proyectos de inversión con alternativas de decisión dicotómicas en el tiempo. En este caso la metodología tradicional castiga la valoración de las posibles ganancias del proyecto, fundamentalmente por que no aprecia las posibilidades potenciales, sino que se concentra en el peso de la incertidumbre.

De esta manera, un proyecto de cualquier ámbito que involucrara etapas de decisión respecto a abandonar el proyecto o no hacerlo, tiene un tratamiento bastante útil con el enfoque de opciones y su valoración. Cada etapa es evaluada en forma separada e incluida en su valuación final.

Concretamente, para el caso de una evaluación de un proyecto de tres fases de inversión y financiamiento, la exposición sería como la que se muestra la Figura 2.

Figura 2. Etapas de un proyecto.

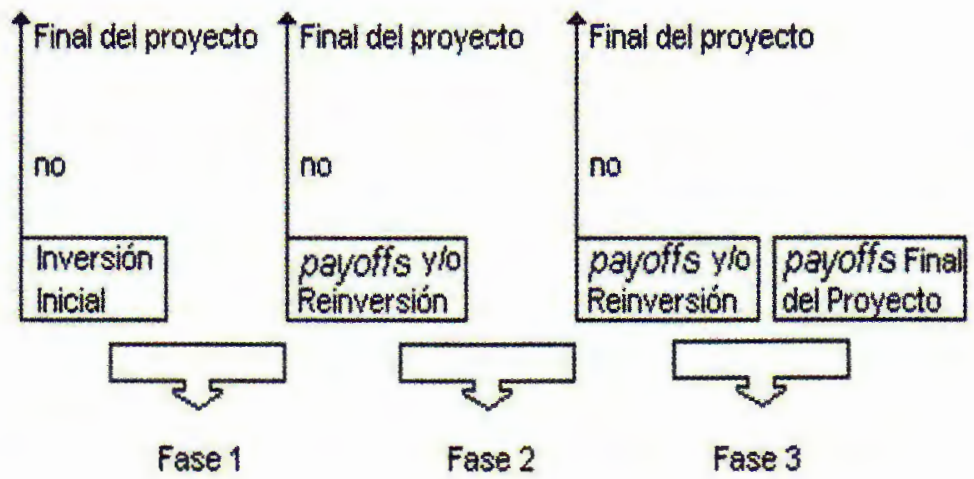

En cada nudo de decisión nos encontramos con la siguiente estructura de juegos. Figura 3.

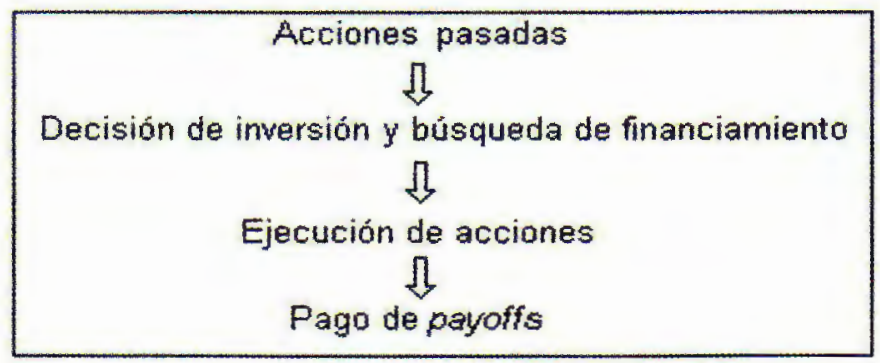

Es claro que cuando se toma la decisión de llevar o no adelante la siguiente etapa del proyecto (o de iniciarlo), todo depende principalmente de los payoffs de cada etapa en particular. Quien decide debe prever las fuentes de financiamiento 
(para luego ir en su búsqueda) y las tareas operacionales antes de tomar la decisión de invertir.

En la valoración del proyecto se consideran los payoffs parciales y a través de éstos la globalidad del mismo, centrándose en la posibilidad de rescindir en cualquier momento inicial de las fases si continúa o no. El equilibrio general final puede desencadenar múltiples alternativas teóricas, pero en cada una de ellas la decisión óptima debe coincidir con un Equilibrio de Nash.

\section{Conclusiones}

Este trabajo presenta un método para analizar problemas de análisis de incertidumbre y valoración de estrategias utilizando la teoría de juegos. Esto se logra con la integración de la teoría de juegos y el option pricing.

El análisis de la teoría de juegos de las opciones complementa a la maximización de utilidad esperada, encontrada en los modelos de la teoría clásica económica. Aun más, el acercamiento de la valoración de opciones tiene la ventaja de considerar el valor del dinero en el tiempo y el precio por el riesgo asumido en forma automática. La principal ventaja del método radica en su habilidad para separar dos problemas económicos: el de la valoración de pagos futuros inciertos y el de las interacciones estratégicas.

En esta integración de enfoques, el análisis de opciones con la teoría de juegos proporciona un eslabón realmente importante que existe entre los mercados y organizaciones: mientras el uso del option pricing permite la valoración de los payoffs de los jugadores, usando el criterio de mercados eficientes, la teoría de juegos toma en cuenta la estructura institucional de las organizaciones y las decisiones que ellas toman.

Sin embargo, el método utilizado en este trabajo tiene algunas limitaciones. Primero, aunque es posible la separación de la valoración y los problemas estratégicos, las expresiones matemáticas obtenidas son bastante complejas. Este obstáculo, que es inherente a la valoración de opciones, significa que simples formas de acercarse a la solución de equilibrio no son siempre posibles.

Segundo, el método sólo trabaja fácilmente si las estrategias óptimas de los jugadores son las no estocásticas, es decir, no dependen del valor tomado por la variable estado. La razón de ello se debe a que si la estrategia óptima es estocástica, la valoración de los payoffs de los jugadores llega a ser insufrible, si no imposible. De esta manera, las estrategias óptimas de los jugadores en las fases precedentes no podrían ser calculadas.

Finalmente, debemos recordar que la modelización en tiempo continuo es una abstracción de la realidad. Por lo tanto, hay que mantener cautela al interpretar los resultados obtenidos. De cualquier modo, todo modelo requiere supuestos restrictivos, y el análisis en tiempo-continuo puede proporcionar una buena aproximación.

\section{Bibliografía}

Agénor, P. R. (2000). Banking Crises: Causes, Effects, and Regulatory Responses. In Macroeconomic Management Financial Stability and Poverty Reduction. World Bank. Sao Paulo, Brazil. pp. 127-140.

Allen, F. and D. Gale (1998). Optimal Financial Crises. Journal of Finance, 53, pp. 12451284 . 
Black, F. and M. Scholes (1973). The Pricing of Options and Corporate Liabilities. Journal of Political Economy, 81(3), pp. 637-659.

Chriss, N. A. (1996). Black Scholes and Beyond: Option Pricing Models. McGraw-Hill Professional Publishing. Chicago.

Constantinides, G. and A. G. Malliaris (2001). Options Markets. Edward Elgar Publishing. Cheltenham.

Damodaran, A. (2002). The Promise and Peril of Real Options. Working Paper. Leonard N. Stern, School of Business, New York.

Dernirguç-Kunt, A. and E. Detragiache (1998). The Determinants of Banking Crises in Developing Countries. IMF, Staff Papers, 45. pp. 81-109.

Eichengreen, B. and A. K. Rose (1998). Staying Afloat when the Wind Shifts: External Factors and Emerging-Market Banking Crises. Centre for Economic Policy Research.

Fernandéz, P. (1996). Opciones, Futuros e intrumentos derivados. Deusto, Bilbao.

Gibbons, R. (1993). Un primer curso de Teoría de Juegos. Antoni Bosch. Barcelona.

Kaminsky, G. (1998). Currency and Banking Crises: The Early Warnings of Distress. International Finance Discussion Papers, Board of Governors of the Federal Reserve System (U.S.), 178.

Kaminsky, G. and C. Reinhart (1999). The Twin Crises: The Causes of Banking and Balance of Payments Problems. Americon Economic Review, 89(3) pp. 473-500.

Lamothe, P. (1993). Opciones financieras: un enfoque fundamental. McGraw Hill.

Martínez, G. X. (2001). Liberalization, Bank Fragility, and Bank Runs in Ecuador. Working Paper, Department of Economics, University of Notre Dame.

Merton, R. C. (1973). Theory of Rational Option Pricing. Bell Journal of Economics and Management Science, 4(1), pp. 141-183.

Paddock, J., D. Siegel, and J. Smith (1988). Option Valuation of Claims on Real Assets: The Case of Offshore Petroleum Leases. The Quarterly Journal of Economics, 103(3), pp. 479-508.

Schwartz, R. and C. W. Smith (1997). Derivatives Handbook-Risk Management and Control. John Wiley \& Sons, Inc. New York.

Tirole, J. (1990). La teoría de la Organización Industrial. Ariel.

Ziegler, A. (1999). A Game Theory Analysis of Options: Contributions to the Theory of Financial Intermediation in Continuous Time. Springer-Verlag. Berlin. 